\title{
Approved nominations to the Fellowship and Membership under Bye-Law III 2 (ii) and approved Affiliates
}

It was agreed that the following candidates should recetve the Fellowship:

Dr M. Abdel-Mawgoud, Dr M. T. Abou-Saleh, Dr D. S. Addala, Dr M. M. M. Al-Mousawt, Dr V. R. Anness, Dr P. R. Aylard, Dr S. M. Bailey, Dr W. A. Barker, Dr H. E. Barnes, Dr K. Barrett, Dr R. Basu, Dr R. N. Bloor, Dr P. J. Bowers, Dr S. Britten, Dr P. W. Brooks, Dr L. M. Brown, Professor A. Burns, Dr L. L. Burton, Dr P. A. Carney, Dr R. R. N. Carvalho, Dr P. M. Chapman, Professor T. A. Cheng, Dr I. Clark, Dr M. G. Clarke, Dr D. A. Cola, Dr R. A. Collacott, Dr D. R. Collyer, Dr J. V. Conway, Dr P. A. Cook, Dr S. J. Cooper, DrS. J. Cope, Dr F. M. Corrigan, Dr. D. I. O. Craufurd, Dr A. Cremona-Barbaro, Dr P. S. Curran, Dr. D. A. Curson, Dr J. M. CurtisRaleigh, Dr O. J. Daniels, Dr T. K. M. Daradkeh. Dr P. B. S. Decalmer, Dr M. S. L. De Silva, Dr V. L. Doughty, Dr L. M. Drummond, Dr J. L. Dunlop, Dr N. L. G. Eastman, Dr D. M. Eminson, Dr. A. I. Etchegoyen, Dr A. F. Francis, Dr D. R. Franks, Dr H. M. Gadhvi, Dr L. Gask, Dr E. A. Gilvarry, Dr S. G. Gowers, Dr P.S. Grahame, Dr J. Greenwood, Dr A. T. Grounds, Dr R. C. Harrington, Dr S. J. Harris, Dr G. L. Harrison, Dr R. M. Hawley, Dr J. A. Hayter, Dr D. T. M. Healey, Dr M. K. Hinchliffe, Dr R. D. Hinshelwood, Dr P. Hoare, Dr F. Holloway, Dr G. W. Hughes, Dr P. M. Hughes, Dr W. C. Hughes, Dr A. V. M. Hughson, Dr S. F. Hung, Dr N. F. S. Hymas, Dr B. W. Jacobs, Dr A. R. Johns, Dr M. J. Kember, Dr C. W. Kenn, Dr R. I. L. Kennedy, Dr E. A. Kerr, Dr R. W. Kerwin, Dr N. I. Kitson, Dr C. A. Lawton, Professor S. W. Lewis, Dr. G. W. Lubby, Dr A. M. Lodge, Dr M. B. Lynch, Dr G. M. P. MacFlynn, Dr J. F. Maguire, Dr F. R. Margison, Dr J. N. Marks, Dr G. Masterton, Dr S. B. Mathur, Dr R. R. McAuley, Dr G. M. G. McClure, Dr H. M. R. Meler, Dr G. Milavic, Dr C. J. Molodynski, Dr P. Moodley, Dr N. Murugananthan, Dr B. N. Nwulu, Dr M. D. O'Brien, Dr S. O'Connor, Dr O. A. Oyebode, Dr S. S. Palia, Dr V. R. Pandita-Gunawardena, Dr D. G. Patterson, Dr. P. E. Potter, Dr A. C. Purches, Dr T. Rajamanickam, Dr M. L. Rennie, Dr E. B. Renvoize, Dr B. L. Roberts, Dr B. A. Robertshaw, Dr C. E. Rowe, Dr S. R. Sadik, Dr W. Y. A. Sarhan, Dr I. G. G. Saunders, Professor J. L. Scott, Dr E. P. Sein, Dr E. Shur, Dr C. Sillince, Dr K. Stvakumar, Dr M. J. Smith, Dr V. J. Spotswood, Dr D. P. Srinivasan, Dr R. E. Steel, Professor A. L. Stein, Dr M. A. Suleiman, Dr A. Theodossiadis, Dr C. P. Treves-Brown, Dr N. W. F. Tyre, Dr P. J. M. Urwin, Dr I. D. Vyas, Dr L. Webster, Dr B. J.
Wells, Dr D. C. Wilkinson, Dr D. J. Will, Dr C. S. Wright, Dr E. Wyn Pugh, Dr K. M. Zaw.

It was agreed that the following candidates should recetve the Membership under Bye-Law III 2 (ii):

Professor C. Hoschl, Dr M. K. Isaac, Professor S. Kasper, Dr K. Kaufman, Dr A. Lindhardt, Professor D. Moussaoui, Dr A. G. Stevens, Dr A. T. Tylee.

The following have been approved as Affiliates of the Royal College of Psychiatrists:

Dr D. H. Abbood, Dr I. E. Abdel Rahman, Dr K. H. Adabie, Dr S. Alfred, Dr J. M. Allatt, Dr N. Anand, Dr I. Ajina, Dr S. L. Axford, Dr M. Azam, Dr S. Banerjee, Dr M. Z. Barsoum, Dr T. M. Basheer, Dr R. Bhageerutty, Dr M. L. Birtwistle, Dr J. M. Bishop, Dr R. J. W. Bolonna, Dr A. Cameron, Dr R. Chart, Dr H. P. S. Chauhan, Dr D. Chavda, Dr A. Choudhury, Dr M. J. Cronin Pozwolskd, Dr J. E. A. Cyrlac, Dr J. M. Darlington, Dr E. J. Davies, Dr N. K. Deb, Dr K. P. De Stlva, Dr V. A. Devi, Dr. B. B. Dhumma, Dr B. B. Dhungana, Dr S. M. Donohue, Dr H. G. G. Dougall, Dr C. M. Downes, Dr A. T. Ekanayake, Dr M. A. El Hihi, Dr S. I. El Kary, Dr K. P. Evans, Dr A. Farsi, Dr K. A. Ferriter, Dr A. B. E. Fox, Dr C. A. H. Foy, Dr J. P. Galway, Dr S. C. Gammie, Dr I. Ghannam, Dr A. B. Gharlb-Omar, Dr R. S. Gill, Dr D. Gnanenthiran, Dr R. D. Gordon, Dr B. Gunasekara, Dr S. M. Gunasekera, Dr A. F. Gunn, Dr M. A. Hamour, Dr A. H. I. Hourani, Dr S. S. Huda, Dr S. A. Hussain, Dr C. Jackson, Dr H. P. Jasenthultyana, Dr K. Jayaratnam, Dr R. S. Jayswal, Dr R. A. Kapuge, Dr B. Kyer, Dr P. S. Kodituwakku, Dr U. Kuber, Dr I. Lakhanpal, Dr M. A. Latif, Dr M. Mackinnon, Dr K. Malik, Dr N. C. Mackenzie, Dr J. L. Majumder, Dr M. Malovic-Yeeles, Dr M. A. McCreadie, Dr S. M. McLean, Dr C. P. Mehta, Dr S. Mehta, Dr M. I. Michail, Dr M-C. A. Michel, Dr C. A. Mitchell, Dr E. Mitford, Dr D. Mitra, Dr M. Mobayed, Dr D. Mukherjee, Dr B. Mukhopadhyay, Dr W. P. Munro, Dr J. E. Muris, Dr P. R. Nandakumar, Dr P. Naveenan, Dr M. G. Orgel, Dr R. Ovtred, Dr R. Pal, Dr V. S. Pandey, Dr P. D. Parghi, Dr P. N. Parlkh, Dr C. S. Patel, Dr. R. Pathmanandam, Dr R. D. Pathak, Dr C. A. M. Perera, Dr M. S. Pleczora, Dr L. Porter, Dr N. Prasad, Dr D. A. 
Provan, Dr M. Quddoos, Dr K. Quinn, Dr M. Qureshi, Dr Y. N. Qureshi, Dr T. Rasamuthiah, Dr S. K. Ray, Dr S. M. Reddy, Dr C. D. Redston, Dr C. C. S. Ridley, Dr D. R. Rimmer, Dr J. L. Romero Urcelay, Dr K. K. Sahota, Dr M. S. Saleswar, Dr F-H. Saleh, Dr L. A. Samarasinghe, Dr D. Santhiapillat, Dr S. F. Sarathchandra, Dr C. E. Segarajasinghe, Dr S. A. Selim, Dr S. Sen, Dr A. K. Sen Gupta, Dr T. Shanmuganathan,
Dr K. K. Shenoy, Dr S. D. Shenoy, Dr P. R. Shetty, Dr Y. P. Shukla, Dr A. M. Siddique, Dr B. Singh, Dr A. N. Singh, Dr M. P. Singh, Dr T. Stvarajasingham, Dr T. Suresh, Dr S. A. Tandon, Dr O. O. M. Thammanna, Dr M. J. Thomas, Dr E. A. Treadwell, Dr A. F. Ullegaddi, Dr R. D. Umaipalan, Dr J. A. Varchevker, Dr R. Veeramani, Dr M. C. Welsh, DrJ. R. Wilkie, DrC.S. Wilson, DrE. M. Wilson, Dr C. E. Yeates, Dr M. G. R. Zakd.

\title{
Management of Violence and Aggression in Health Care
}

\author{
Edited by Brian Kidd and Cameron Stark
}

A multidisciplinary approach to the management of violence, bringing together expert knowledge from medical, nursing, social work and other professionals

$\square$ Describes ways of assuaging violence both practically and with the use of medication

$\square$ Discusses the role of the organisation and ethical considerations

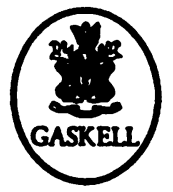

\section{- $112.50 \bigcirc 200 p p . \odot 1995 \bigcirc$ ISBN 0902241842}

Available from bookshops and from the Publications Department, Royal College of Psychiatrists, 17 Belgrave Square,

London SW1X 8PG (Tel. 0171-235 2351 extension 146) 